\title{
Reasons for using inhalants: Evidence for discrete classes in a sample of incarcerated adolescents
}

\author{
Brian E. Perron, (Ph.D.) $)^{\mathrm{a}, *}$, Michael G. Vaughn, (Ph.D. $)^{\mathrm{b}}$, Matthew O. Howard, (Ph.D.) ${ }^{\mathrm{a}}$ \\ ${ }^{a}$ University of Michigan, Social Work, Ann Arbor, MI 48109-1106, USA \\ ${ }^{\mathrm{b}}$ University of Pittsburgh, Pittsburgh, PA 15260
}

Received 28 February 2007; received in revised form 26 April 2007; accepted 22 May 2007

\begin{abstract}
The use and abuse of inhalants continue to be among the most pernicious, curious, and least understood of drug use behaviors. The purpose of this study was to identify specific subgroups of adolescents based on their reasons for using inhalants. Data regarding reasons for using inhalants were examined using latent profile analysis. A three-class solution exhibited the best fit with the data. Although validation analysis revealed that the classes did not differ on demographic characteristics, classes exhibited significant differences across numerous clinical measures, including anxiety, problems associated with using substances, global symptom severity, and number of different types of inhalants used. Results indicate heterogeneity for reasons for use of inhalants and associated psychological distress. Findings may provide clues to future taxonomic development of inhalant abuse phenomena and prevention. (C) 2008 Elsevier Inc. All rights reserved.
\end{abstract}

Keywords: Inhalants; Substance abuse; Latent profile analysis; Adolescent drug use

\section{Introduction}

Studies indicate that an estimated $9 \%$ to $20 \%$ of adolescents have engaged in "huffing" or "sniffing" of inhalants such as gasoline, glue, shoe polish, paint, and correction fluid (Garofalo, Wolf, Wissow, Woods, \& Goodman, 1999; Johnston, O’Malley, Bachman, \& Schulenberg, 2006; Wu, Pilowsky, \& Schlenger, 2004). Effects are rapid and include slurred speech, ataxia, and other symptoms that are similar to alcohol intoxication (Rosenberg \& Sharp, 1997). The inhaling of volatile organic compounds contained within these widely available household products among adolescents remains poorly understood. Although there is speculation on why people use inhalants, to our knowledge, there has not been any systematic study of the reasons for inhalant use. Brouette and Anton (2001) also argue that, while the rate of inhalant abuse continues to rise,

* Corresponding author. University of Michigan, Social Work, 1080 South University Avenue, Ann Arbor, MI 48109-1106, USA. Tel.: +1 314 2557262.

E-mail address: beperron@wustl.edu (B.E. Perron). it is still one of the least studied or discussed groups of abused substances. This is unfortunate because knowledge of the reasons for using inhalants may eventually lend itself to the development of targeted prevention and treatment strategies. Different reasons for use may also shed light on the types of youth who abuse inhalants and their associated levels of psychopathology. If there is heterogeneity in reasons for use that are distinctly associated with various indicators of internalizing and externalizing behaviors, then this knowledge not only can stimulate future taxonomic research on inhalant use but also can focus treatment resources.

The purpose of this study was to explore the existence of relatively discrete classes of youth based on reasons for using inhalants by employing latent profile analysis (LPA). Classes identified were then compared across measures of psychological distress, externalizing behaviors, and frequency of inhalant use. As this is the first study to utilize latent variable modeling to uncover homogeneous subgroups of adolescent inhalant users, we had no a priori taxonomy from which to derive classes. Thus, no hypotheses are offered. 


\section{Materials and methods}

\subsection{Sample and procedures}

The present study sample $(N=277)$ of inhalant users was drawn from a larger survey of the population of current residents $(N=740)$ in the Missouri Division of Youth Services (DYS). Participation in the study was voluntary. The Missouri DYS is the legal guardian of all residents who are committed to its care by the state's 45 juvenile courts. Face-to-face interviews of all youth were conducted using a comprehensive solvent assessment inventory. All interviewers completed an intensive 1-day training session, and an interview editor was on-site at each facility as youth were interviewed to minimize interviewer omissions and errors. DYS residents are under 24-hour-a-day supervision; thus, interviews were conducted in large rooms that provided private areas where confidential interviews could be conducted simultaneously with between three and six youth. The sample recruitment protocol ensured that no youths who had completed the interview at one facility attempted to complete or were successful in completing the interview at another facility. This study was approved by DYS, the Washington University Human Studies Committee Institutional Review Board, and the federal Office of Human Research Protection and was granted a Certificate of Confidentiality by the National Institute on Drug Abuse. Youth received US\$10.00 for their participation.

All youth providing written informed consent completed the structured, face-to-face, approximately 45-minute interview assessing demographic characteristics, lifetime and annual use of inhalants, other drug use, substance-related problems, current and lifetime psychiatric symptoms, and externalizing behaviors.

\subsection{Survey}

A set of 15 survey items reflecting a broad range of reasons for using inhalants was included in the survey. These items were adapted from the Reasons for Drinking Questionnaire (RFDQ; Zywiak, Connors, Maiso, \& Westerberg, 1996). Subjects responded to the survey items by indicating their level of agreement for use based on a 5-point Likert-type response scale $(1=$ strongly disagree, 5 = strongly agree). These survey items exhibited good face validity with respect to inhalant use. The internal consistency of the items was $\alpha=.82$. Because this is a new measure, no additional information about its psychometric properties is available.

\subsection{Analytic strategy}

\subsubsection{Latent profile analysis}

As previously stated, LPA was used to identify subgroups of subjects who provided qualitatively different sets of reasons for using inhalants. LPA is similar to latent class analysis (LCA), except that LPA uses continuous observed measures whereas LCA analyzes dichotomous measures. The underlying assumption of LPA is that the relationship among continuous indicators can be explained by a categorical latent variable. The continuous indicators are considered to be locally independent, meaning that the observed items are statistically independent within each latent class (Lazarfeld \& Henry, 1968; Ruscio, 2004).

LPA was carried out using Mplus (version 4.2) in an exploratory fashion. Specifically, rather than testing a class solution specified a priori, the fit of a series of different models was examined. A single-class model was examined first, and classes were added until no further improvements were observed. The empirical fit of the model was based on the Bayesian Information Criterion (BIC). An improvement in model fit results in a lower value on the BIC. Entropy was also examined, which is an index reflecting how well the indicators predict class membership. Values closer to 1.0 indicate better prediction.

Because there are a number of models that can exhibit a good fit with the data, the conceptual fit of the model was also considered in the analysis. This involved examining the overall results and diagnostics of the model and using visual representations of class profiles to assess the interpretability and practical implications of class solutions.

\subsubsection{Validation analysis}

A validation analysis of the final model was conducted by testing associations of class characteristics with demographic and clinical measures. This analysis was carried out using SPSS (version 14.0). Measures were selected based on prior empirical associations with substance use among adolescents.

Specific demographic measures included age (in years), gender (male/female), and receipt of public assistance (yes/ no). Because DYS system youth are predominantly African American and White, race was measured by collapsing categories to create a dichotomous measure (White/nonWhite). Location of residence prior to commitment was measured with four categories: urban city, suburban area, small town, and rural area.

A set of clinical measures was selected for the validation analysis. These included four measures from the Brief Symptom Index (BSI), namely, depression, anxiety, paranoid ideation, and psychoticism, and the overall global severity sum score $(\alpha=.96)$. Prior experiences of suicide ideation and substance use problems were assessed using the Massachusetts Youth Screening Inventory (i.e., MAYSI-2). These subscales consist of yes/no items. The total number of affirmative item responses was then summed to provide an overall scale score. Studies using the MAYSI-2 in incarcerated youth samples have found it to be reliable (Grisso, Barnum, Fletcher, Cauffman, \& Peuschold, 2001). Polysubstance use was derived from a composite of selfreported use episodes of 14 types of substances. A measure of externalizing behaviors was derived from the Self-Report 
of Delinquency $(\alpha=.84)$, which asks youth if they have been involved in various forms of nonviolent and violent antisocial acts in the past 12 months. From a set of 65 different types of inhalants, subjects were also queried about the different types of inhalants they used over their lifetime. It should be noted that higher scores on the clinical measures represent more problems or a higher degree of clinical severity.

\section{Results}

\subsection{Sample characteristics}

Approximately $75 \%$ of the sample $(n=209)$ was White, and $84 \%(n=233)$ were male. The mean age was 15.6 years $(S D=1.12)$. Thirty-nine percent $(n=107)$ reported that their family received public assistance. We examined differences between inhalant users and noninhalant users using chi-square and $t$ tests. No differences were observed with respect to age, gender, and family receipt of public assistance. However, inhalant use was associated with urbanicity, $\chi^{2}(1)=53.72, p<.001$, with a greater proportion of inhalants living in small towns/ urban areas. Inhalant use was also more common among White subjects versus non-White subjects, $\chi^{2}(1)=131.83$, $p<.001$.

\subsection{Descriptive summary}

Table 1 summarizes the reasons, ordered by the highest mean value of agreement. Curiosity about the effects of inhalants was the most common reason for using inhalants, followed by feelings of boredom and that they are easier to get than other drugs. Liking inhalants more than other drugs was the least common answer. Feeling angry with someone else, angry with self, and family problems also had low mean values.

Table 1

Summary of reasons for using inhalants $(N=277)$

\begin{tabular}{lll}
\hline Reason & $M$ & $S D$ \\
\hline Curious about effects & 2.76 & 1.07 \\
Feeling bored & 2.38 & 1.27 \\
Easier to get than other drugs & 2.25 & 1.41 \\
Fun to use & 2.03 & 1.33 \\
To relax & 1.89 & 1.28 \\
Not dangerous & 1.82 & 1.30 \\
To forget about troubles & 1.69 & 1.40 \\
Peer pressure & 1.60 & 1.37 \\
Feeling sad & 1.38 & 1.30 \\
Feeling anxious & 1.29 & 1.21 \\
To impress others & 1.26 & 1.23 \\
Angry at someone & 1.24 & 1.26 \\
Family problems & 1.23 & 1.29 \\
Angry at self & 1.10 & 1.17 \\
Like more than other drugs & 0.92 & 1.05 \\
\hline
\end{tabular}

Table 2

Empirical fit of models and class sizes based on LPA

\begin{tabular}{llll}
\hline Class solution & BIC & Entropy & Class size, $n(\%)$ \\
\hline 1 Class & $13,861.47$ & NA & $277(100)$ \\
2 Classes & $13,027.28$ & .925 & $169(61.8)$ \\
& & & $108(39.2)$ \\
3 Classes & $12,766.44$ & .903 & $85(30.6)$ \\
& & & $122(44.1)$ \\
4 Classes & & $70(25.3)$ \\
& & .902 & $61(22.0)$ \\
& & & $76(27.6)$ \\
5 Classes & & $86(31.1)$ \\
& $12,725.30$ & & $53(19.3)$ \\
& & $77(27.7)$ \\
& & $50(17.9)$ \\
& & $69(24.9)$ \\
& & $50(18.2)$ \\
\end{tabular}

\subsection{Latent profile analysis}

A total of five LPA models were examined, ranging from one to five classes. Each model was estimated with 200 random starts, and no problems with local maxima were observed. The empirical fit of the models and their estimated class sizes are summarized in Table 2. The oneclass solution exhibited a poor fit with the data relative to the other models. A decrease in the BIC was observed with the subsequent models, with the exception of the five-class solution, which showed a slight increase relative to the fourclass solution. The entropy values for all the models were very similar and greater than .90 , indicating that the reasons for using inhalants were strong predictors of class membership. However, the differences in entropy values across the different solutions were not considered to have any practical significance. Overall, the four-class solution exhibited the best empirical fit with the data based on the BIC. However, the improvement from the three-class to four-class solution was very small.

The conceptual fit of the models was examined through visual inspection. This involved plotting the estimated mean values for each reason by each class. The four-class solution was examined first. Although each class of this model was clearly distinguishable, Classes 2 and 3 had mean values that were nearly identical for 8 of the 15 reasons. For the seven reasons that showed noticeable differences, Class 3 exhibited higher values. The differences in these two classes did not appear to have practical significance.

The profiles of the three-class solution were clearly distinguished (see Fig. 1). There was also greater variability in mean values on the reasons compared to the four-class solution. The class sizes were also fairly balanced (see Table 2). Class 1 had the lowest mean agreement values for all the reasons. Although the overall fit of the three-class solution was slightly lower than that of the four-class solution based on the BIC, this model was considered to have greater conceptual fit and practical implications. Thus, the three-class solution was retained as the final model. 


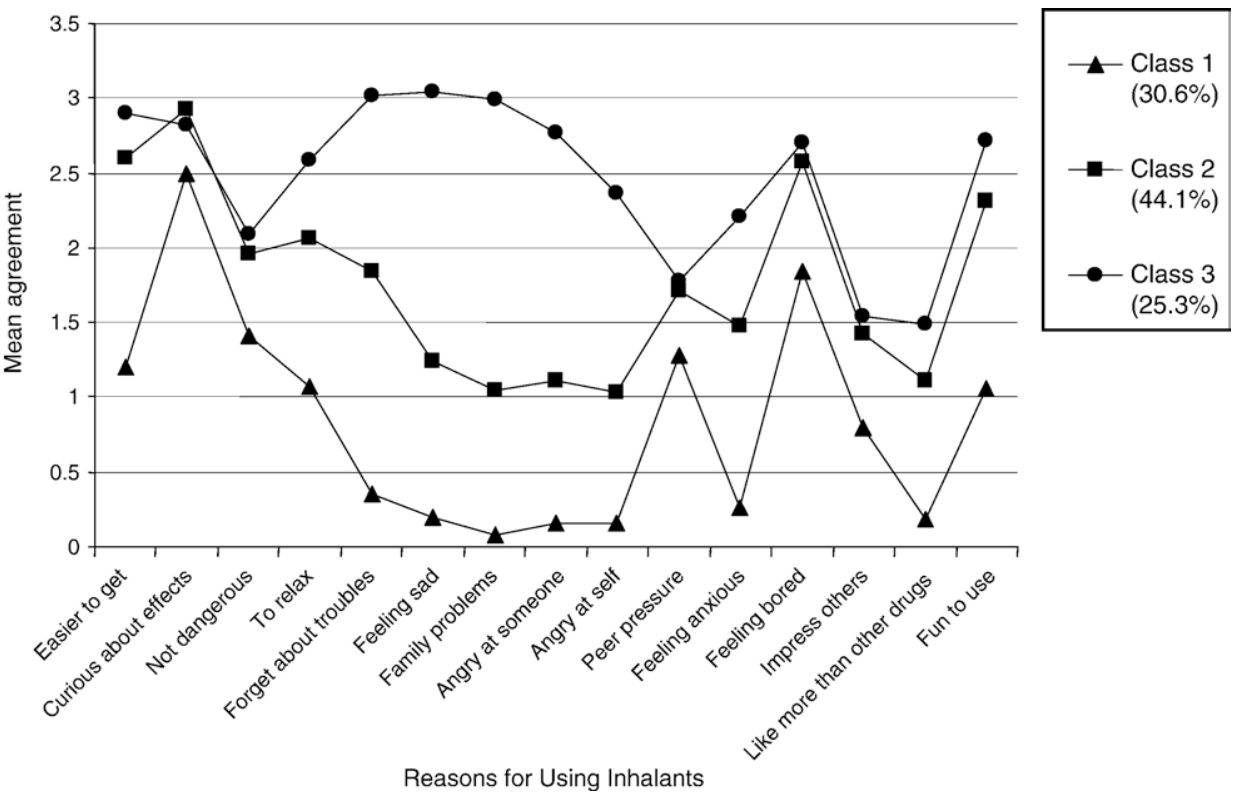

Fig. 1. Three-class solution representing mean agreement on reasons for using inhalants.

\subsection{Validation analysis}

A validation of the three-class solution was conducted by examining the associations among the three classes with demographic and clinical variables. Chi-square tests revealed that class composition did not differ by race, $\chi^{2}(2)=5.58, p=.06$, gender, $\chi^{2}(2)=2.32, p=.313$, receipt of public assistance, $\chi^{2}(2)=1.74, p=.42$, or urbanicity, $\chi^{2}(6)=5.85, p=.44$. One-way analysis of variance (ANOVA) showed that class composition was not associated with age, $F(2)=0.014, p=.99$.

ANOVA with Dunnett's T3 post hoc comparisons revealed significant differences on all the clinical variables (see Table 3). On the Substance Use Problems Index, all three groups were significantly different. Classes 1 and 3 exhibited mean values that were different from Class 3 on all clinical measures. The mean values of Classes 1 and 2 exhibited significant differences on just the Substance Use Problems Index. Class 1 had the lowest mean values on all the measures, and the abusing class had lower mean values than the dependence class. The strongest effect sizes observed, based on $\eta^{2}$, were on the Substance Use Problems Index $\left(\eta^{2}=.13\right)$, Anxiety-BSI $\left(\eta^{2}=.14\right)$, and the Global Severity Index $\left(\eta^{2}=.13\right)$.

Cohen (1988) suggested that a zero-order correlation of .30 was a medium effect size. Thus, the proportion of shared variance $\left(r^{2}\right)$ of this effect is .09 . This provides the basis for comparing the $\eta^{2}$ values, suggesting that the effect sizes in these analyses are of moderate strength and of clinical significance.

The number of different types of inhalants used ranged from 1 to 16. This was a count variable; hence, it exhibited a high skew $(M=4.18, S D=3.04$, skew $=1.05)$. Thus, it was dichotomized, reflecting one and more than one type of inhalant used over the lifetime. The classes showed significant differences on this measure, $\chi^{2}(2)=$ 10.6, $p=.005, \Phi=.21$. Thirty-eight percent of Class 1 subjects used more than one type of inhalant over the lifetime; among Class 2 subjects, 76\%; and among Class 3 subjects, $86 \%$.

Table 3

Tests of association among three-class solution and clinical variables using ANOVA $(N=277)$

\begin{tabular}{|c|c|c|c|c|c|}
\hline Variables & Class $1, M(S D)$ & Class $2, M(S D)$ & Class $3, M(S D)$ & $F$ statistic $^{\mathrm{a}, \mathrm{b}, \mathrm{c}}$ & $p\left(\eta^{2}\right)$ \\
\hline Suicidality Index & $2.86(2.43)$ & $2.98(2.41)$ & $4.27(2.21)$ & $8.42^{\mathrm{b}, \mathrm{c}}$ & $<.001(.06)$ \\
\hline Substance Use Problems Index & $4.14(2.35)$ & $5.31(1.94)$ & $6.24(.159)$ & $21.34^{\mathrm{a}, \mathrm{b}, \mathrm{c}}$ & $<.001(.13)$ \\
\hline Depression (BSI) & $4.73(5.27)$ & $5.49(4.70)$ & $8.91(6.38)$ & $13.25^{\mathrm{b}, \mathrm{c}}$ & $<.001(.09)$ \\
\hline Anxiety (BSI) & $4.16(4.39)$ & $4.90(4.18)$ & $9.03(6.30)$ & $22.30^{\mathrm{b}, \mathrm{c}}$ & $<.001(.14)$ \\
\hline Paranoid ideation (BSI) & $6.36(4.77)$ & $6.59(4.44)$ & $9.29(4.69)$ & $9.64^{\mathrm{b}, \mathrm{c}}$ & $<.001(.07)$ \\
\hline Psychoticism (BSI) & $3.39(3.69)$ & $4.29(4.07)$ & $6.87(5.04)$ & $13.82^{\mathrm{b}, \mathrm{c}}$ & $<.001(.09)$ \\
\hline Global Severity Index & $42.98(32.84)$ & $48.52(30.38)$ & $76.71(43.58)$ & $20.54^{\mathrm{b}, \mathrm{c}}$ & $<.001(.13)$ \\
\hline Externalizing behaviors & $23.14(16.05)$ & $27.41(19.50)$ & $33.06(19.67)$ & $5.41^{\mathrm{b}}$ & $<.001(.04)$ \\
\hline Polysubstance use & $34.02(21.84)$ & $36.16(19.57)$ & $42.67(22.60)$ & $3.46^{\mathrm{b}}$ & $.03(.02)$ \\
\hline
\end{tabular}

Note. Dunnett's post hoc comparisons conducted for all ANOVAs: $\mathrm{a}=$ Classes 1 and 2 are different; $\mathrm{b}=\mathrm{Classes} 1$ and 3 are different; $\mathrm{c}=\mathrm{Classes} 2$ and 3 are different. 


\section{Discussion}

Before reviewing the main findings of this study, it is important to recognize the study limitations. Most important, these data are representative of only incarcerated adolescents. As an enriched sample, these youth typically have a considerable history of substance use and psychiatric problems, which makes it difficult to determine the extent to which the classes and their associations with clinical variables are associated with use of inhalants. Additionally, there are differential system responses to adolescent offending behavior - that is, some enter the juvenile justice system directly, whereas others may be diverted to psychiatric systems of care or adjudicated directly back to the community. Thus, the demographic and clinical characteristics of this study sample may differ substantially from adolescents drawn from community-based samples. Although the reasons for using inhalants were adapted from an existing measure (i.e., RFDQ), the psychometric properties of the measure in this study are not completely known. Therefore, the results should be interpreted with caution.

Curiosity about the effects of inhalants, feelings of boredom, and peer pressure were the major features of Class 1. Members of this class also exhibited the lowest mean value on the Substance Use Problems Index and had a smaller proportion of class members using only one type of inhalant compared to the other classes. Although data on frequency of use were not available, these data provide some evidence that members of Class 1 were "experimental" users.

Members of Class 2 showed consistently higher mean values than members of Class 1 on all the reasons for use. A distinguishing feature of Class 2 was that inhalants were used because members considered them easier to get. The higher mean values for all the reasons, access to inhalants, and greater proportion of using multiple inhalants suggest that these adolescents can be regarded as "active" users.

Compared to Class 2, Class 3 exhibited similar pattern of reasons for using with the exception of reasons related to interpersonal problems and affective symptoms. Specifically, members of this class were using to forget about troubles, to assuage feelings of sadness, to deal with family problems, and because they felt angry with someone else. These members were clearly using inhalants to cope with various types of distress. The proportion of subjects in Class 3 using multiple inhalants was significantly higher than the proportion of subjects in Classes 1 and 2. Thus, the members of this class can be regarded as "coping/highdistress" inhalant users. The consistent associations with clinical variables also support this, with members of Class 3 exhibiting significantly greater severity scores than Class 1 on all these measures.

It should be noted that Class 1 members displayed the lowest levels of clinical severity on all the measures in relation to Class 3. However, there was very little differentiation of Class 2 from Class 1. Specifically, only one clinical variable, substance use severity, was associated with a significant difference across these classes. Although they exhibited unique reasons for use, a broader set of clinical differences across the classes is needed to validate this class structure. Moreover, the foregoing analysis of class structure and associations with clinical variables does not disentangle any particular causal mechanisms. While the reasons for use studied may explain the differential pattern of inhalant use and consequently manifest levels of psychopathology, it is also possible that the psychopathology and history of the individual influence the expressed reasons for use. It is also possible that there is a complex feedback relationship at play.

Longitudinal data, which were not available for this study, would be especially useful to reveal whether classes differed in their use of inhalants over time. A longitudinal analysis can also help reveal whether adolescent inhalant users move in a stage-wise process from experimentation to a substance use disorder. Future taxonomic research on inhalant users should attempt to study the stability of identified classes across time, employ diagnostic mental health inventories, and assess the moderating effects of situational influences, family problems, and inhalant use and other drug use progression. Future research should also attempt to integrate reasons for using with desistance from inhalants and other drugs (e.g., Titus, Godley, \& White, 2006).

Findings reveal that curiosity about inhalant use is high in both the univariate summary and LPA. This finding may possess clear service implications with respect to preventative efforts (e.g., social marketing). It may also be possible to develop targeted treatment approaches, such as using the expressed reasons as a guide in cognitive-behavioral or motivational interviewing. For example, members of the abusers/high-distress class (Class 3 ) reported using inhalants to cope with a variety of problems. Treatment professionals could guide these individuals in systematically assessing the effectiveness and consequences of such behaviors and explore other alternatives.

\section{Acknowledgment}

Funding for this study was provided by NIDA Grants No. DA 15929 and DA 15556; NIDA had no further role in study design; in the collection, analysis, and interpretation of data; in the writing of the report; or in the decision to submit the paper for publication.

\section{References}

Brouette, R., \& Anton, R. (2001). Clinical review of inhalants. American Journal of Addictions, 10, 79-94.

Cohen, J. (1988). Statistical power analysis for the behavioral sciences. (2nd ed.). Hillsdale: Lawrence Erlbaum. 
Garofalo, R., Wolf, R. C., Wissow, L. S., Woods, E. R., \& Goodman, E. (1999). Sexual orientation and risk of suicide attempts among a representative sample of youths. Archives of Pediatrics and Adolescent Medicine, 153, 487-493.

Grisso, T., Barnum, R., Fletcher, K. E., Cauffman, E., \& Peuschold, D. (2001). Massachusetts youth screening instrument for mental health needs of juvenile justice youths. Journal of the American Academy of Child and Adolescent Psychiatry, 40, 409-418.

Johnston, L. D., O’Malley, P. M., Bachman, J. G., \& Schulenberg, J. E. (2006). Monitoring the future national survey results on drug use, 1975-2005: Volume I, secondary school students (NIH Publication No. 06-5883). Bethesda, MD: National Institute of Drug Abuse.

Lazarfeld, P. F., \& Henry, N. W. (1968). Latent structure analysis. Boston: Houghton Mifflin.
Rosenberg, N. L., \& Sharp, C. W. (1997). Solvent toxicity: A neurological focus. Substance Use and Misuse, 32, 1859-1864.

Ruscio, J. (2004). A nontechnical introduction to the taxometric methods. Understanding Statistics, 3, 151-194.

Titus, J. C., Godley, S. H., \& White, M. K. (2006). A post-treatment examination of adolescents' reasons for starting, quitting, and continuing the use of drugs and alcohol. Journal of Adolescent Substance Abuse, 16, 31-49.

Wu, L., Pilowsky, D. J., \& Schlenger, W. E. (2004). Inhalant abuse and dependence among adolescents in the United States. Journal of the American Academy of Child and Adolescent Psychiatry, 43, 1206-1214.

Zywiak, W. H., Connors, G. J., Maisto, S. A., \& Westerberg, V. S. (1996). Relapse research and the Reasons for Drinking Questionnaire: A factor analysis of Marlatt's relapse taxonomy. Addiction, 91, 121S-130S. 